\title{
Adhesion to Non-Sticky Materials
}

An interdisciplinary research team at Kiel University (CAU) has developed a new technology that is capable of joining materials with a low surface energy such as PFTE and silicone. The technology uses nano-scaled crystal linkers as internal „staples“, thus opening up solutions to a large number of technical challenges, for example in medical engineering.

$6 \mathbf{6}$ If the nano staples make even extreme polymers like Teflon and silicone stick to each other, they can join all kinds of other plastic materials", said Professor Rainer Adelung, who is leading the „Functional Nano Materials" group at the Institute of Materials Science in Kiel (CAU) and leads the research project from the materials science side. The new technology of joining materials without chemical modifications can be used, according to Adelung, in a variety of everyday life and high-tech applications. The technique is easy to use and does not need expensive equipment or material.

The „staples“ are micro and nano scaled crystals made of zinc oxide. They are shaped like tetrapods, with four rigid legs protruding from the point of origin. Large-scale tetrapods are known for their ability to interlock and form strong bonds, and are used for example as wave-breakers in coastal protection on the islands of Sylt or Helgoland.

\section{The adhesive principle}

To join the polymers, the zinc oxide crystals are sprinkled evenly onto a heated layer of Teflon. A layer of silicone is then poured on top. In order to join the materials, they are heated to $100{ }^{\circ} \mathrm{C}$ for less than an hour. "It's like stapling two nonsticky materials from the inside," explained Xin Jin, the first author of the publication, who is currently working on her PhD thesis in Kiel. Her colleague and supervisor, Dr. Yogendra Kumar Mishra, explains the adhesive principle: "If you try to pull out a tetrapod on one arm from a polymer layer, the shape of the tetrapod will simply cause three arms to dig in deeper and to hold on even more firmly."
In high-tech businesses such as medical engineering, there is a strong demand for innovative ways to make polymers, particularly silicone, stick to other materials, for example for breathing masks, implants or even small sensors. Such medical applications require materials that are absolutely nonharmful, in other words biocompatible. Many joining methods involve chemical reactions: the materials are glued together. However, the chemical processes may change the polymers' properties and in extreme cases may even make them toxic. By contrast, the tetrapod stapling is a purely mechanical process, which leads the Kiel research team to assume it to be biocompatible. With the tetrapod staples, the silicone bond withstands a load of 200 Newtons per metre. This is similar to peeling conventional sticky tape off a glass surface. "The stickiness we have achieved with the nano tetrapods is remarkable, because, as far as we could verify, no one has ever made silicone and Teflon stick to each other at all", said Lars Heepe, PhD student from the Zoological Institute of Kiel University, who precisely measured the adhesion and analysed the stapled material on a microscopic scale.

\section{Function by switching}

Three research groups combined their expertise in material science, chemistry and biomechanics in this study within the Collaborative Research Center 677 "Function by Switching" (CRC 677). The results are fed directly into both practical applications and further fundamental research. The local business partner nanoproofed $\mathrm{GmbH}$ is already developing a product for painting on top of silicone. In the special research field, the staples are also the basis for developing biomimetic adhesives, for which adhesion can be switched on and off by light

A detailed analysis of the results was presented on 24 August in the journal Advanced Materials.

For further information, please contact: Christian-Albrechts-Universität Kiel, Prof. Rainer Adelung, Institute of Materials Science, ra@tf.uni-kiel.de

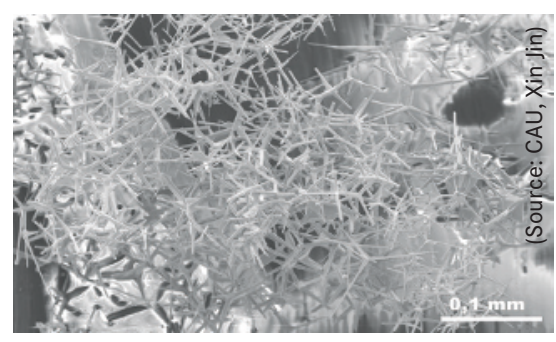

The arms of the tetrapod crystal staples interlock with each other forming strong bonds (image from scanning electron microscope)

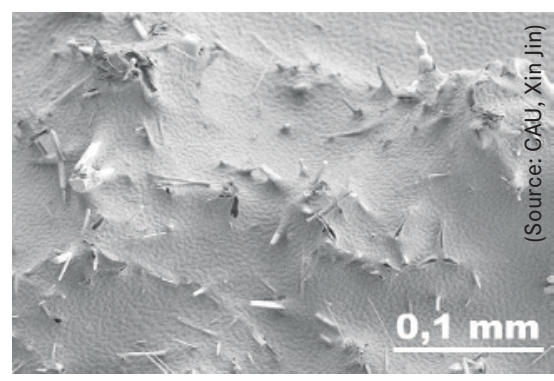

The tetrapod staples sink into the polymer, while some of the arms stick out, acting as hooks.

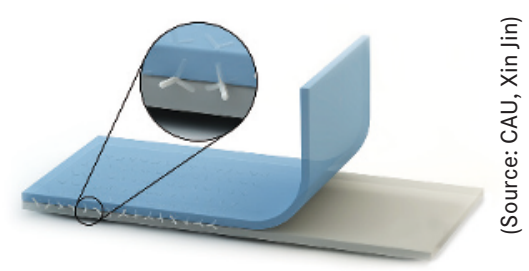

Two polymer layers are stapled from the inside using tetrapod nano staples (concept drawing) 